\title{
Proceso de ejecución de chimeneas industriales de ladrillo valencianas y murcianas
}

\section{Building process of industrial chimneys of brick in Valencia and Murcia}

\author{
$\underline{\text { G. López Patiño }}^{(*)}$, P. Verdejo Gimeno ${ }^{(*)}$, A. Martínez Antón ${ }^{(*)}$, J. Benlloch Marco ${ }^{(*)}$
}

\section{RESUMEN}

A partir de entrevistas realizadas a distintos maestros constructores de chimeneas industriales de ladrillo, pertenecientes a las escuelas valenciana y murciana, que recurren a estilos y sistemas de ejecución muy diferentes, se ha procedido a describir los sistemas empleados en España para la construcción de estos monolitos industriales.

Los sistemas abordados implementan los conocimientos de la Historia de la Construcción española, con un elemento que tuvo una corta vida, pero que fue esencial en la evolución industrial y son clave a la hora de enfrentarnos a una posible restauración del elemento.

Palabras clave: Chimenea industrial; ladrillo; construcción; ejecución; patrimonio industrial; memoria del trabajo.

\section{ABSTRACT}

The system used in Spain for the construction of industrial brick chimneys in east and south east of Spain and those that have been its influence is described in this paper, from interviews with different master builders belonging to the Valencia and Murcia schools, with styles and systems of very different execution.

The addressed systems implement the knowledge of the Spanish Construction History, of an item that had a short life though it was essential in industrial development and becomes the key for a possible restoration of the element.

Keywords: Industrial chimney; brick; construction; execution; industrial heritage; work memory.

${ }^{(*)}$ Universitat Politècnica de València (Valencia, España).

Persona de contacto/Corresponding author: glopez@csa.upv.es (G. López Patiño)

Cómo citar este artículo/Citation: López Patiño, G., Verdejo Gimeno, P., Martínez Antón, A., Benlloch Marco, J. (2016). Proceso de ejecución de chimeneas industriales de ladrillo valencianas y murcianas. Informes de la Construcción, 68(543): e153, doi: http://dx.doi. org/10.3989/ic.15.068.

Licencia / License: Salvo indicación contraria, todos los contenidos de la edición electrónica de Informes de la Construcción se distribuyen bajo una licencia de uso y distribución Creative Commons Attribution License (CC BY) Spain 3.o. 


\section{INTRODUCCIÓN}

Una chimenea industrial de ladrillo es una construcción esbelta, hueca, de sección transversal decreciente conforme se aumenta la altura, realizada en material cerámico en su práctica totalidad, cuyas funciones están relacionadas con fines higienistas favoreciendo la dispersión del humo a la atmósfera, así como mejorar la combustión gracias al tiro que produce la diferencia de densidades del aire caliente generado en el interior y el aire frío del exterior.

Tres son la partes visibles de una chimenea (Figura 1), cada una con características propias, tanto funcionales, como estéticas y constructivas, y que, desde la parte inferior hasta la superior, son: (a) la base situada sobre la cimentación, que aporta estabilidad sobre el terreno siendo, por lo general, más ancha que el resto de elementos, provista en la mayor parte de los casos de un podio y rematada por una cornisa en voladizo; (b) el fuste, tubo o caña, con pendiente continua en el exterior, pero con escalones distantes en el interior como apuntan Valdés (1) y Rebolledo (2), y (c) la corona que remata, cuya forma tiene una influencia perceptible en aumentar o disminuir el tiro cuando el viento sopla (3). Aunque tipológicamente pueden realizarse varias clasificaciones, la más usual es la que aporta información sobre la resistencia al viento, basada en la forma de la sección transversal del fuste. En un principio esta sección fue cuadrada, evolución directa de la chimenea doméstica, para pasar a la forma ideal con menor resistencia que es la circular. Según Buchanan (4) la presión que ejercía el viento en una sección cuadrada era el
$33 \%$ superior a la ejercida sobre una sección circular. Entre estas pueden encontrarse las secciones octogonales y, en menor medida, singulares fustes de sección hexagonal.

La dimensión de una chimenea varía dependiendo de la capacidad de la máquina de vapor a la cual iba asociada. En nuestro país, la media de altura de chimeneas de ladrillo está en los $25 \mathrm{~m}$.

Las manifestaciones recogidas a agentes intervinientes en su proceso de ejecución, desde promotores a maestros de obras contratados originarios del levante y sureste español y su área de influencia, han sacado a la luz la práctica de un oficio reconocido, y ya perdido en nuestros días, en el que los constructores simultaneaban la práctica de la albañilería, base de los trabajos, con el diseño singular de estas elevaciones fabriles llevadas a cabo después de la Guerra Civil española, hasta bien entrada la década de los sesenta del siglo xx. Paralelamente, se consideraban extintas las chimeneas de palastro mientras que las grandes industrias erigían elevados pináculos de hormigón, fuera del alcance técnico y económico de la pequeña empresa de período post-bélico.

El estudio establece, sin menoscabo de otras posibles escuelas en el territorio español, la escuela valenciana y murciana de construcción de chimeneas industriales de ladrillo (5), atendiendo a características de los tipos construidos, con fustes octogonales en el primer caso y fustes de sección circular en el segundo, así como a sistemas utilizados en la ejecución de las mismas detallados a continuación (Tabla 1).

Tabla 1. Cuadro resumen de las características más importantes de los constructores murcianos, valencianos e influencias.

\begin{tabular}{|c|c|c|c|c|c|c|}
\hline Procedencia & Constructor & $\begin{array}{c}\text { Sección } \\
\text { fuste }\end{array}$ & Aparejo & $\begin{array}{c}\text { Pendiente } \\
\text { (\%) }\end{array}$ & Dosificación & Andamios \\
\hline $\begin{array}{l}\text { Comunidad } \\
\text { Valenciana }\end{array}$ & Fam. Goig & Octogonal & Inglés & 2,5 & $1 \mathrm{cal}: 3$ arena & $\begin{array}{l}\text { Cada } 7 \text { hiladas. } \\
\text { Con escala de gat }\end{array}$ \\
\hline $\begin{array}{l}\text { Comunidad } \\
\text { Valenciana }\end{array}$ & A. Martínez & Octogonal & Inglés & & $1 \mathrm{cal}: 2$ arena & En cruz \\
\hline $\begin{array}{l}\text { Comunidad } \\
\text { Valenciana }\end{array}$ & Fam. Mir & Octogonal & Inglés & $2-2,5$ & $\begin{array}{l}2 \text { cal : } 3 \text { arena } \\
6 \text { cal }: 1 \text { cemento }\end{array}$ & \\
\hline $\begin{array}{l}\text { Comunidad } \\
\text { Valenciana }\end{array}$ & Fam. Alfonso & Octogonal & Inglés & $1-1,5$ & $\begin{array}{l}4 \text { cal : } 10 \text { arena, parte superior: } \\
1 \text { capazo cemento }\end{array}$ & \\
\hline $\begin{array}{l}\text { Comunidad } \\
\text { Valenciana }\end{array}$ & Crespo & Octogonal & Inglés & 2,5 & 2-3 cal :10-12 arena : 1 cemento & $\begin{array}{l}\text { Cada } 80 \mathrm{~cm} \text { o } 17 \\
\text { hiladas }\end{array}$ \\
\hline $\begin{array}{l}\text { Comunidad } \\
\text { Valenciana }\end{array}$ & V. Ramírez & Octogonal & Inglés & 2,5 & 5 cal :10 arena : 1 cemento & \\
\hline $\begin{array}{l}\text { Comunidad } \\
\text { Valenciana }\end{array}$ & Cortés & Octogonal & Inglés & 2,5 & $1 \mathrm{cal}: 4$ arena & \\
\hline $\begin{array}{l}\text { Comunidad } \\
\text { Valenciana }\end{array}$ & A. Reig & Octogonal & & 2,5 & & \\
\hline $\begin{array}{l}\text { Comunidad } \\
\text { Valenciana }\end{array}$ & Jareño & $\begin{array}{l}\text { Octogonal y } \\
\text { hexagonal }\end{array}$ & Inglés & $2-2,5^{-} 3$ & $\begin{array}{l}2 \text { cal : } 3 \text { arena } \\
12 \text { espuertas mortero : } 1 \text { cemento }\end{array}$ & Cada 9 hiladas \\
\hline $\begin{array}{l}\text { Comunidad } \\
\text { Valenciana }\end{array}$ & Serrano & Octogonal & Inglés & 2 & $1 \mathrm{cal}: 2$ arena & \\
\hline $\begin{array}{l}\text { Comunidad } \\
\text { Murciana }\end{array}$ & $\begin{array}{l}\text { Riquelme y equipo } \\
\text { de Alcantarilla }\end{array}$ & Circular & Flamenco & 2,5 & 2 cal : 3 arena & Cada 7-8 hiladas \\
\hline $\begin{array}{l}\text { Comunidad } \\
\text { Murciana }\end{array}$ & Alcañiz & Circular & Flamenco & 2,5 & $1 \mathrm{cal}: 3$ arena & \\
\hline $\begin{array}{l}\text { Comunidad } \\
\text { Murciana }\end{array}$ & José Miñana & Circular & Flamenco & 2,5 & $\begin{array}{l}1 \text { cal : } 1 \text { arena, cemento para base } \\
\text { no para caña }\end{array}$ & \\
\hline $\begin{array}{l}\text { Comunidad } \\
\text { Murciana }\end{array}$ & $\begin{array}{l}\text { Equipo de } \\
\text { Millas (con fam. } \\
\text { Menchero) }\end{array}$ & Circular & $\begin{array}{l}\text { Con cuñas } \\
\text { a tizón }\end{array}$ & 2,5 & $\begin{array}{l}1 \text { cal : } 3 \text { arena, cada 6-7 medidas } \\
\text { una de cemento }\end{array}$ & Cada 6 hiladas \\
\hline
\end{tabular}




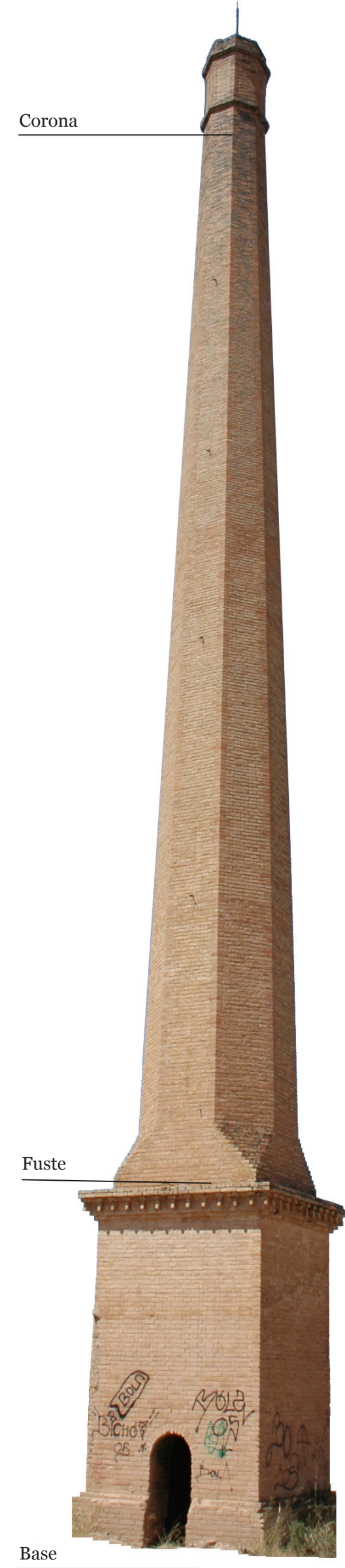

Figura 1. Chimenea de Casinos (Valencia).

\section{PROCESO DE EJECUCIÓN}

\subsection{Organización del trabajo: época y personal}

Bancroft y Bancroft (6) aconsejan el trabajo de construcción de chimeneas con temperaturas cálidas que eviten congelaciones, para facilitar el completo fraguado y adquisición de resistencias del mortero. Sin embargo, constructores entrevistados manifiestan haber construido con temperaturas gélidas no sólo en el levante sino también en el interior de la península. La desestabilización es fácil en lo alto de la construcción fabril debido al movimiento que producen el empuje del viento y el tiro producido, y por ello paralizaban el trabajo hasta que cesaba la oscilación. Por motivos de seguridad se abandonaba también el puesto de trabajo cuando eran sorprendidos por tormentas eléctricas.

Mientras los constructores valencianos confían el trabajo de ejecución de la chimenea en cinco personas (Figura 2): dos oficiales, o un oficial y ayudante de oficial, que trabajan en la parte superior colocando ladrillos, un peón por debajo de ellos que realiza el acopio de material en altura y aporta el mismo a los oficiales que están en el andamio por encima del suyo, y otros dos peones abajo que manejan el torno de la polea o la cuerda misma de esa polea, y el amasado del mortero, atado de ladrillos, llenado de capazos, etc.; para los murcianos el número mínimo de trabajadores es de ocho, divididos en dos oficiales arriba, uno se encarga de repartir el mortero y el otro coloca ladrillos, un peón junto a ellos encargándose del acopio de material, otro peón abajo manejando la polea, dos amasando y otros dos preparando el material para transportarlo en vertical. El personal de oficiales suele aportarlos el constructor y la peonada era proporcionada por el promotor.

Aunque no era práctica habitual, ante la dificultad que supone la altura, los ruidos circundantes, el viento y el tiro para el mantenimiento de una buena comunicación, se manejó un lenguaje de signos por parte de algunos constructores. Los gestos más usuales entre los murcianos eran mostrar el brazo extendido con el puño cerrado para que se subiese masa $^{1}$, y el brazo extendido con la mano abierta para pedir cuñas; es decir, piezas cerámicas en forma de sección de corona circular.

El tiempo medio estimado para la construcción de una chimenea es de mes a mes y medio, dependiendo de la altura y sección de la chimenea, el número de personas que trabajen, lo concienzudo del trabajo, los sistemas, etc. Así pues, aparecerán construcciones en las que se emplearán de dos a tres meses, como las realizadas por una de las familias más prolíficas en la construcción de chimeneas, los alcireños Goig, o incluso por debajo del mes, como los 23 días empleados por Jareño para la realización de la chimenea de P. Vela en Quintanar de la Orden.

\subsection{Diseño y cálculo del modelo}

El diseño de una chimenea va dirigido a conseguir la altura suficiente para conseguir la máxima eficacia del tiro, conjugando los aspectos estructurales de la estabilidad, por encima de la estética. Por ello, los primeros ingenieros manejaban

\footnotetext{
${ }^{1}$ Se refiere así el constructor para aludir al mortero.
} 


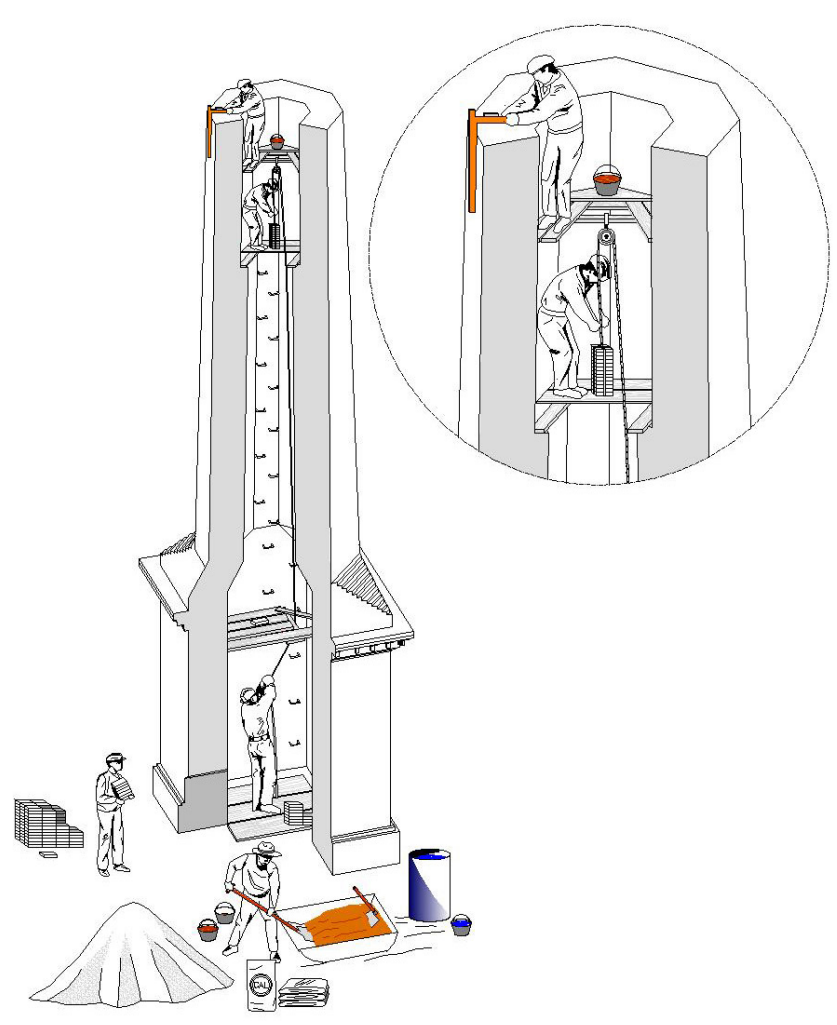

Figura 2. Equipo de trabajo valenciano.

variables como el tipo de combustible, el área del fuego y la longitud del conducto hasta las calderas, aunque estas premisas no siempre han sido respetadas.

En general, los constructores estudiados, sin participación de técnicos con mayor cualificación, dibujaban la chimenea en el suelo para calcular las dimensiones de su base y fuste, así como calcular el número de ladrillos necesario para organizar los pedidos de material.

Para realizar este primer cálculo se comienza por la boquilla. El espacio interior debía ser suficiente para que un trabajador pudiera estar en la cumbre colocando ladrillos, así que, esta dimensión variaba según los estándares de comodidad de los constructores. Lógicamente, esta dimensión perfila la estilización de la figura de la chimenea, afectando a su estética. Se procede a ejemplificar un cálculo con la dimensión utilizada por los valencianos Goig para los que el diámetro mínimo interior en boquilla para una chimenea de $30 \mathrm{~m}$ era de $80 \mathrm{~cm}$, siendo el espesor de la boquilla de un pie. Considerando que el espesor ha de contabilizarse por duplicado, la dimensión exterior mínima será:

$$
0,80 \mathrm{~m}+0,25 \times 2=1,30 \mathrm{~m}
$$

Teniendo en cuenta que para la familia Goig 16 hiladas corresponden a $1 \mathrm{~m}$ de altura, y que la corona tiene $32 \mathrm{hi}-$ ladas contadas, la dimensión de la misma es de $2 \mathrm{~m}$, que se descuentan para el cálculo de la dimensión de la base. También José Riquelme, de la saga murciana, refiere este hecho de los $2 \mathrm{~m}$ de la corona, y del descuento para el cálculo final.

La dimensión media en altura de las bases está comprendida entre 3 y 5 m, que en este caso, carente de pendiente, o del $5 \%$, según los datos aportados. Este hecho, junto con el del párrafo anterior, supone que tenga que descontarse de la altura total de la chimenea a efectos de ajustar la pendiente:

$$
30 \mathrm{~m} \text { (altura total) }-2 \mathrm{~m}(\text { corona })-3 \mathrm{~m}(\text { base })=25 \mathrm{~m}
$$

Seguidamente se calcula la dimensión que corresponde por altura la pendiente aplicada. Si la pendiente es del 2,5\%, teniendo en cuenta que son dos secciones longitudinales:

$$
25 \mathrm{~m} \times(2 \times 2,5 \%)=1,25 \mathrm{~m}
$$

Sumándole la dimensión exterior en boquilla a esta cantidad obtenemos la dimensión exterior en la base del fuste:

$$
1,30 \mathrm{~m}+1,25 \mathrm{~m}=2,55 \mathrm{~m}
$$

Para una chimenea octogonal esa sería también la dimensión del cuerpo de la base, aunque para la cornisa puede aumentarse medio o un pie. Para una chimenea de sección de fuste circular se suele añadir unos o,30 m más a cada lado, con lo que la dimensión del lado del cuadrado de la cara superior de la base aumenta finalmente 0,60 m más. Esto supone que, como la boquilla murciana es un poco más pequeña, la dimensión de la base sería de 3,05 m.

Si a esto se le añade la pendiente, si la tuviera, que para bases octogonales es del $5 \%$, y para base ejecutadas por murcianos entre el 2,5 y el $5 \%$, tendríamos finalmente la dimensión de la base. Debe recordarse que la pendiente ofrecida para el fuste puede variar aunque en general se aplica el 2,5\%.

El hueco practicado para la cimentación es cúbico, con independencia de la sección de la base, y su dimensión en planta suele tener $1 \mathrm{~m}$ más por cada lado de la base.

\subsection{Preparación de materiales}

Tiempo antes de la llegada de los oficiales comenzaban los preparativos de los materiales, principalmente la cal, empleada como conglomerante base del mortero. Se aprecia el uso de distintos procedimientos y tiempos a la hora de apagar la cal viva, aérea, en terrón. El tiempo de espera para el uso de la cal, justificado por la mayor adquisición de plasticidad de la misma, variaba desde un par de meses, cubriéndola para evitar su endurecimiento prematuro, hasta sólo un par de días, manteniendo la precaución de cribar primero los granos que no se habían apagado para evitar la posterior expansión en la puesta en obra.

Otra operación habitual previa a la ejecución de la chimenea consistía en la humectación de los ladrillos, aunque Mir declara no sumergir los ladrillos en agua para evitar el hinchamiento de los caliches de los mismos. También Bancroft y Bancroft (6) se refieren al empapado de ladrillos antes de llevar a cabo los trabajos de fábrica.

\subsection{Transporte de materiales}

Los materiales eran suministrados básicamente por el interior del fuste de la chimenea. En un principio, para las grandes chimeneas, se utilizaba la energía animal girando alrededor de un torno enrollando o desenrollando cuerda y, más tarde, se recurrió al motor a vapor situado exteriormente al pie del pedestal (7). 
Para el accionamiento de la polea valenciana y murciana se empleó exclusivamente la fuerza humana, bien directamente con la cuerda o a través de un torno.

La polea utilizada por equipos murcianos estaría compuesta de dos discos ranurados unidos mediante pletina metálica de $1 \mathrm{~m}$ aproximadamente formando una anilla, unidos a su vez a una estructura de madera, formada por un madero de unos $3 \mathrm{~m}$ de longitud y escuadría de $8 \times 8 \mathrm{~cm}$ rigidizada con una escuadra, también de madera (Figura 3). Esta estructura lígnea se introduce en al menos 3 anillas de la escala interior, descansando en el tercer escalón mediante un pasador, donde se fija con ayuda de cuerdas y cuñas de madera, de manera que una de las poleas queda en el exterior de la chimenea, por donde se recibirá el material de construcción, ladrillos y mortero, y la otra quedará situada en el hueco interior, de manera que uno de los extremos de la cuerda circula por el interior de la chimenea, mientras que el otro, por donde se izan los materiales queda por el exterior. La cuerda exterior termina en una anilla de donde pende un gancho del que se cuelga un cubo con asa de anilla cerrada, para izar los materiales. A esa misma anilla se engancha otra cuerda para evitar que el cubo pueda balancearse y golpear o rozar la construcción (7), así como ayudar al deslizamiento descendente cuando el cubo está vacío y el peso propio no es suficiente. En todo momento está manejada desde la parte inferior de la chimenea.

El sistema de polea valenciana consistía en un solo disco ranurado por donde discurre una cuerda. Esta polea pende de

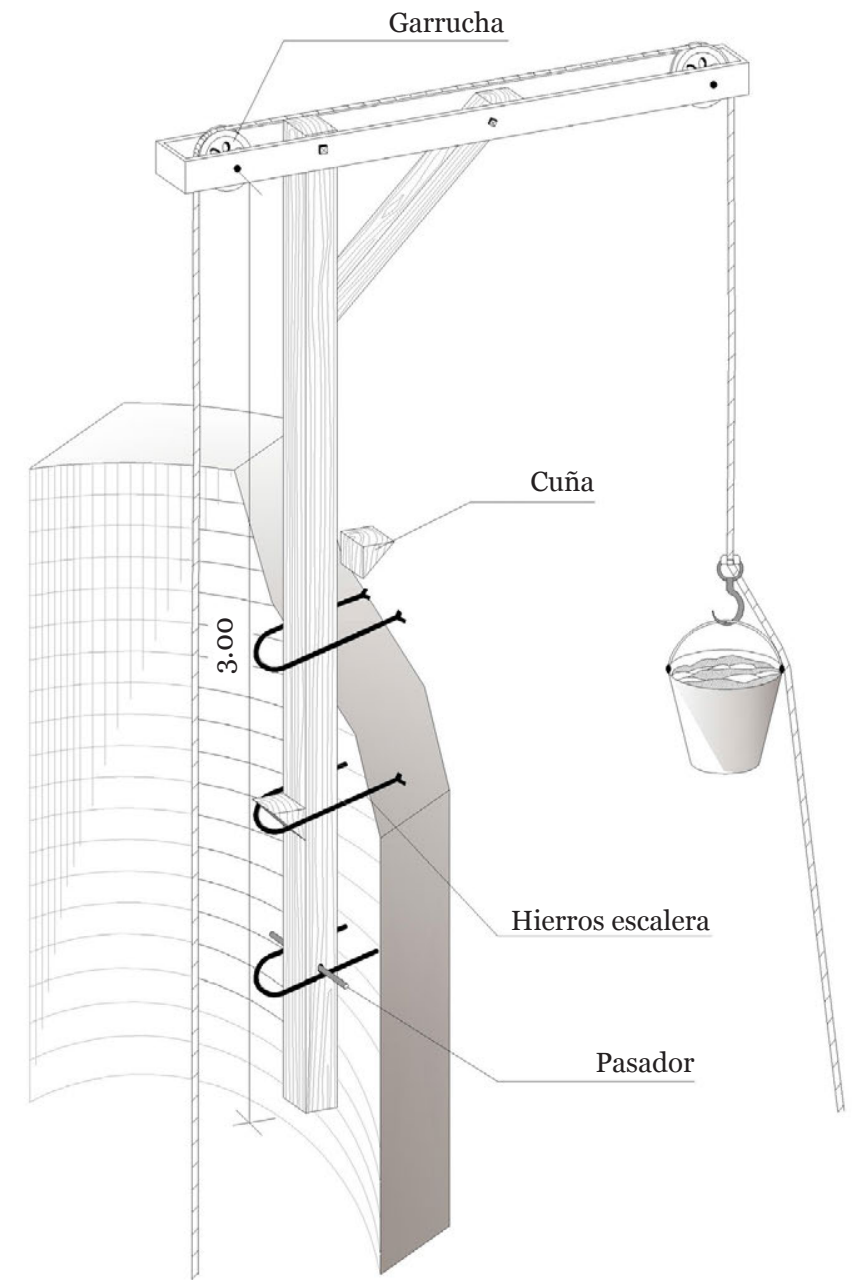

Figura 3. Sistema de colocación de polea murciana. un madero, que forma parte del andamio. La mayoría de las veces se sitúa en un lateral para facilitar la ascensión de materiales, aunque otras queda en el centro del hueco interior de la chimenea.

Todos los constructores entrevistados coinciden en el número de ladrillos transportados de una vez que es de cinco, atados con trenilla o en una gaveta. Cada dos o tres envíos de ladrillo se realiza uno de mortero.

\subsection{Ejecución de la fábrica}

La cimentación estaba completamente terminada cuando llegaban los oficiales a construir la chimenea, según declara Bernardo Pérez, oficial y familiar del alcireño Agustín Goig, a pesar de la documentación contractual encontrada donde se especifica que la cimentación correrá a cargo del constructor.

Riquelme, perteneciente a la saga murciana de los Pacheco, reseña que la cimentación se realiza con hormigón y piedra maceada, hasta llegar a la rasante del terreno, dejando un hueco central de unos 70-90 cm de lado para el aposadero de humos, también llamado cenicero, para recoger las cenizas. Esta cavidad no era ejecutada de forma generalizada, como refiere el constructor Salvador Mir.

Para asegurar la verticalidad del eje de la chimenea, así como la regularidad de circularidad de cada hilada alrededor de su centro, De las Rivas recurre al cintrel, que consiste en lo siguiente:

...se principia por trazar la base del fuste sobre el plano de coronamiento del pedestal de donde arranca la primera hilada, valiéndose de dos reglas de madera, una de longitud igual al diámetro exterior y la otra algo más larga que el radio exterior; la primera está provista en su punto medio de una punta que se hace corresponder con el eje de la chimenea; la segunda lleva practicado un taladro en uno de sus extremos, dispuesto para que pueda girar alrededor de la punta de la primera; a partir del taladro se fijan dos puntas a distancia de los radios interior y exterior, que sirven para describir las dos circunferencias de la corona, sección de la base del fuste. Esta regla recibe el nombre de cintrel... (8).

La realización de mechinales para apoyar los andamios interiores se ejecutaba eliminando una pieza a tizón, en dos hiladas, quedando un hueco de dimensiones cúbicas. Algunas chimeneas muestran estos mechinales en dos caras enfrentadas, y para no mermar sección y resistencias, se procedía en el siguiente nivel de andamiaje a la realización de huecos en caras opuestas.

La transición de base cuadrada a fuste octogonal, de difícil resolución en su ejecución y aparejo, se realiza mediante pechinas escalonadas (Figura 4). Estas pechinas pueden dejarse vistas con el escalonado, o rellenarse para dar el aspecto de superficie completamente lisa e incluso curvada (Figura 5). Diversas son las maneras de ejecutar esta esquina; primeramente se procede a levantar varias hiladas con la misma dimensión que el cuerpo de la base o un poco menos, lógicamente con la misma sección cuadrada de la base, a la que se va aplicando ya la pendiente. En realidad el número de hiladas va a variar dependiendo del número de hiladas acordada para el escalonado. 


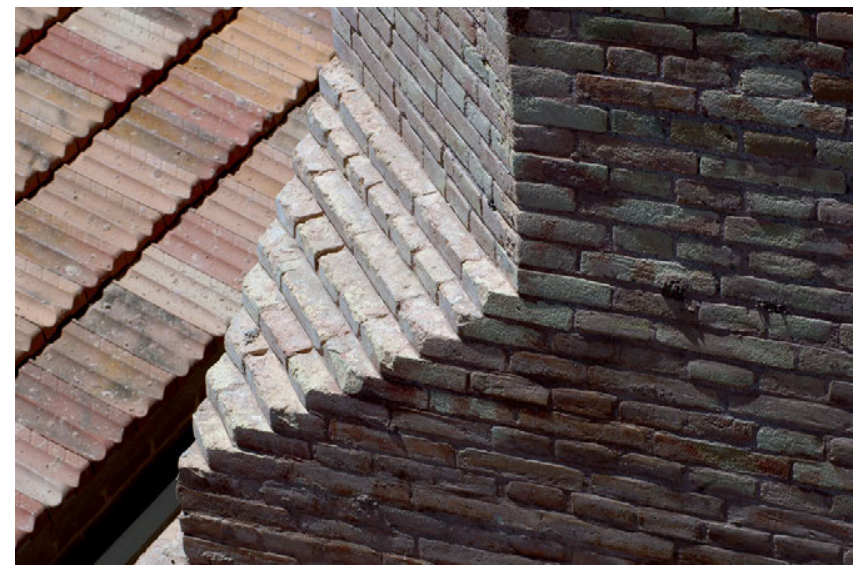

Figura 4. Pechina escalonada.

Para chimeneas de envergadura se han contabilizado cinco ladrillos colocados a tizón, más las piezas de las dos esquinas correspondientes a ese lado del octógono, en el arranque del fuste. En chimeneas de menor entidad los ladrillos a tizón disminuyen hasta tres. Cuando la chimenea es muy pequeña, se pierde esta razón. Se ha observado que la primera pieza que se coloca para conseguir el lado puede ser una pieza especial que recoge la forma de toda la esquina, o bien puede utilizarse una pieza aplantillada esquinera. En la segunda hilada siempre van dos piezas de esquina unidas por su lado más corto (Figura 6). Para un resultado homogéneo, el lado corto

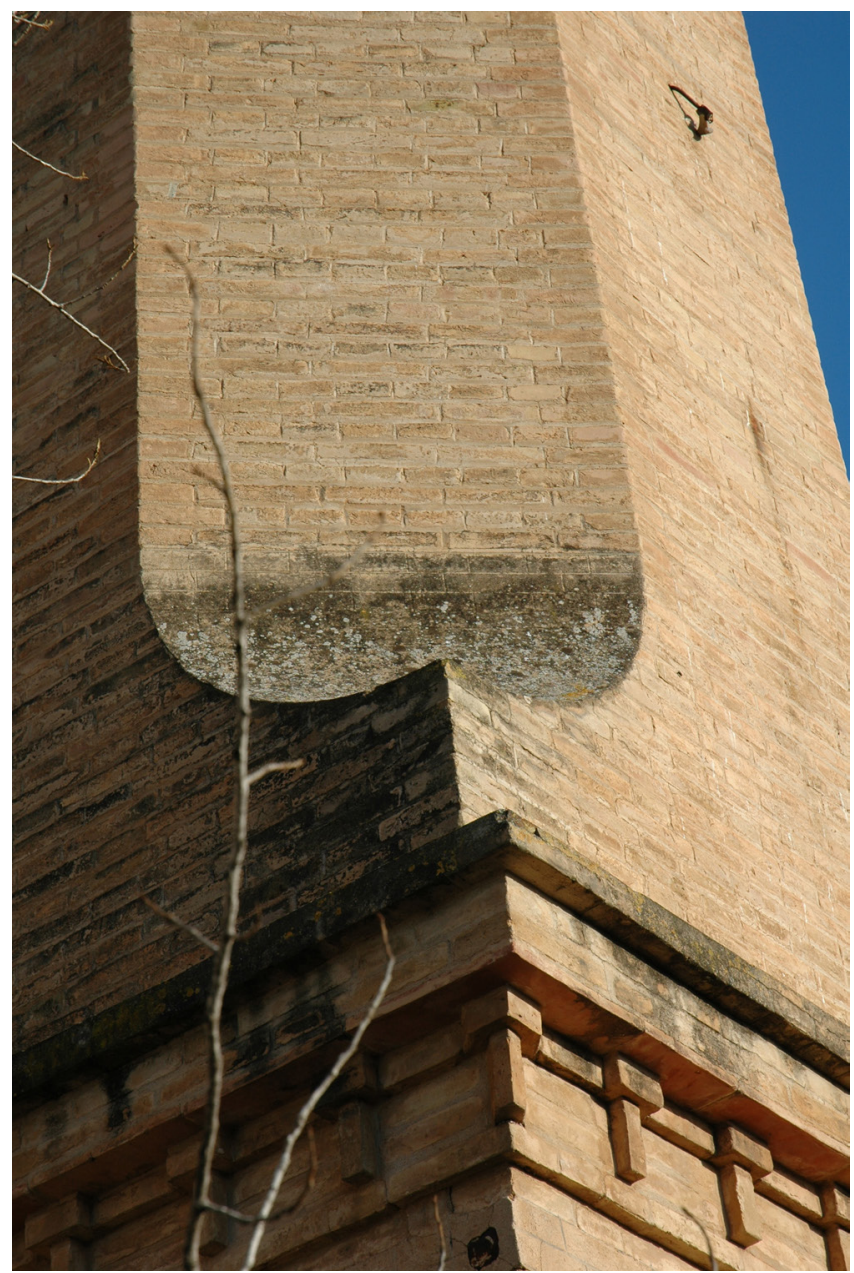

Figura 5. Pechina curvada. Chimenea fábrica del hielo L'Eliana (Valencia). de la pieza de esquina se coloca en el escalonado que va ganando el lado del octógono, mientras que la parte larga queda siempre en el lado que va perdiendo en favor del que gana.

La colocación de ladrillos se lleva a cabo siempre en el mismo sentido, indiferente si es horario o antihorario. El oficial más experimentado coloca las piezas de esquina, cuando se trata de chimenea de sección poligonal, con ladrillos aplantillados especiales que forman el ángulo del polígono. El otro oficial va rellenando el hueco dejado entre piezas de ladrillo aplantillado. Cuando el primero termina de colocar todas las esquinas procede igualmente al relleno, de manera que, mientras el segundo oficial ejecuta unas cinco caras, el primero ejecuta tres caras y las esquinas. En cuanto al aparejo, este se pierde del exterior al interior en función del espesor de la fábrica, permitiendo que cuando la parte vista de la hilada está ejecutada a tizón, la parte interior queda vista a soga, consecuente con la traba existente.

Jareño, manchego de Tomelloso, plantea un sistema diferente a sus maestros Goig, como consecuencia directa de la prescindibilidad del taulaplom, utensilio de madera utilizado para comprobar la pendiente. Para ello monta un andamio exterior con 4 postes de madera y crucetas, colocando tablas que servirán de base para una plantilla de madera. La altura del andamio será de $3 \mathrm{~m}$ por encima de la base. Dicha plantilla tendrá un tamaño un poco superior al tamaño del círculo donde está inscrito el polígono determinado para la sección del fuste, a esa altura. Se practican unos taladros en la plantilla en el lugar que correspondería a la esquinas. Se introduce una cuerda por el taladro con una plomada en el extremo y se deja caer hasta que el plomo llega a la parte superior de la base. Se abren entonces las cuerdas la distancia en centímetros correspondiente según altura y pendiente, y se fijan las mismas. Luego sólo hay que colocar las piezas siguiendo la cuerda. Como la pendiente es continua, una vez se ha obtenido la experiencia suficiente en los $3 \mathrm{~m}$, se desmonta plantilla y andamio y se sigue sin ayuda de referencia alguna.

El de Tomelloso empleará un sistema parecido al descrito por De las Rivas para asegurar verticalidad y centralidad de las secciones de la chimenea. En el centro del cenicero se realiza una marca para saber cuál va a ser el centro de las secciones del fuste, y se clava un clavo que servirá para comprobar los centros de esos círculos. Cuando se realiza la comprobación de verticalidad se coloca una tabla con la medida del diámetro de la hilada a comprobar con un agujero en el centro. Se introduce una cuerda con una plomada y se deja caer, para cerciorarse de la coincidencia de la punta de la plomada con el clavo. Con otra cuerda se traza entonces la circunferencia para comprobar el abollamiento de la forma circular (Figura 7).

En el caso de la ejecución de chimeneas de fuste circular, se puede proceder para grandes diámetros con piezas paralepipédicas trabajadas a soga, nunca a tizón, pues las trabas son más difíciles de ejecutar. Para pequeños diámetros se utilizarán ladrillos colocados a tizón, o ladrillos aplantillados en cuña, aunque este sistema repercute en la traba. La ejecución de hiladas de este tipo de sección con cuñas exige la colocación, en primer lugar, de toda la periferia con las cuñas de mayor tamaño, y luego el relleno posterior con piezas más pequeñas que decrecen con el diámetro.

Para chimeneas de grandes dimensiones o varios colocadores, las directrices vienen determinadas para evitar diferen- 

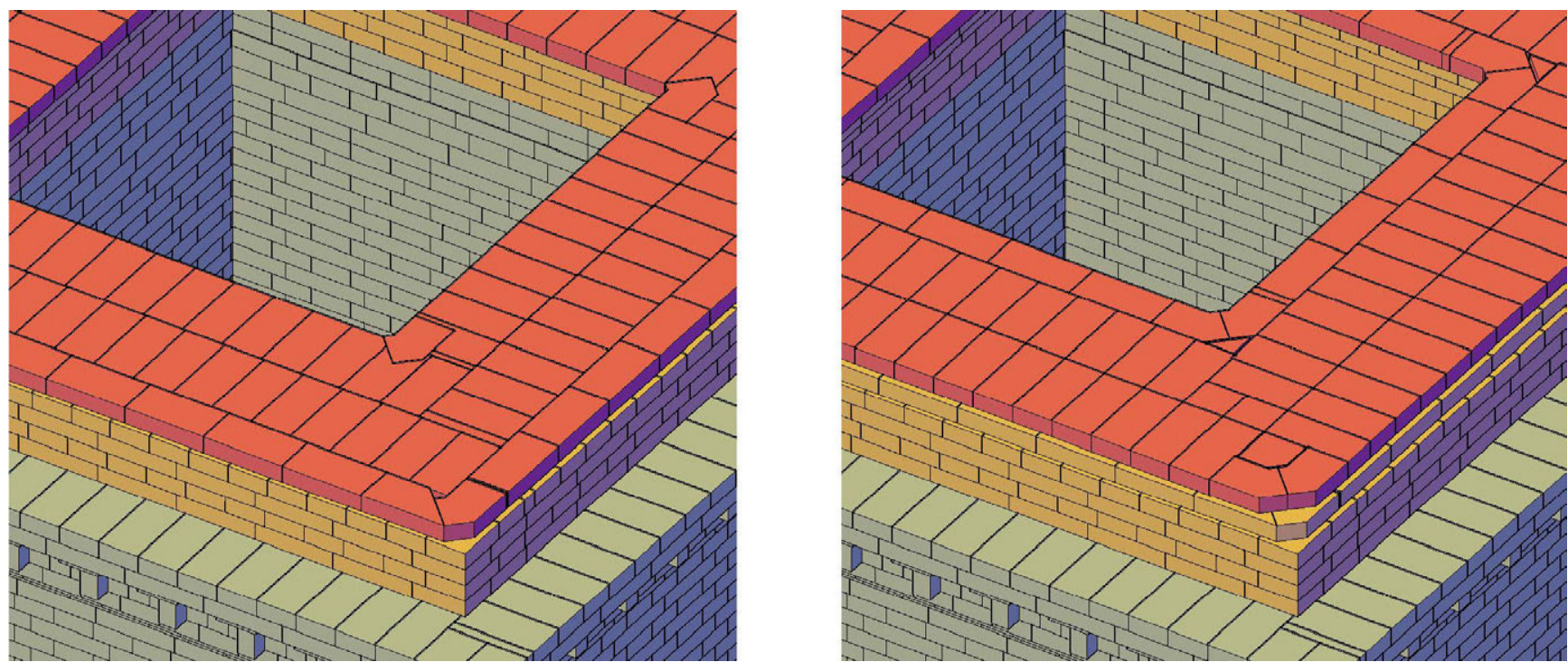

Figura 6. Ejecución de primera y segunda hilada de pechina escalonada.

cias notables y apreciables en la ejecución de la fábrica, así que todos los colocadores recorrerán todo el perímetro horizontal del fuste en toda su longitud. En las chimeneas de fuste circular la precaución debe ser máxima pues el resultado es más evidente.

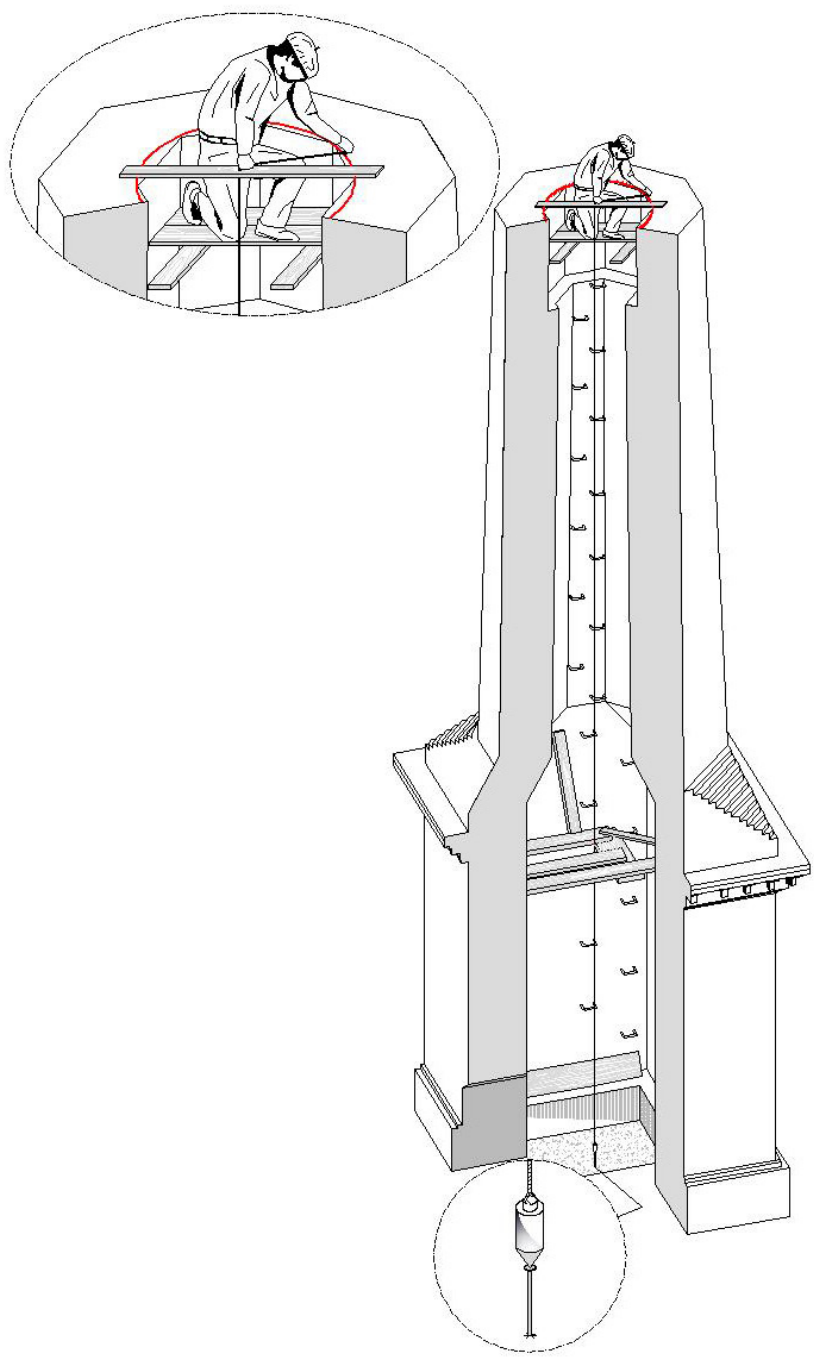

Figura 7. Comprobación de la circularidad de la sección.
El mortero se extiende por toda la superficie de la última hilada. Los ladrillos se colocan a restregón. Conforme se gana en altura se aumenta la cantidad de cemento en el mortero bastardo.

Para una correcta ejecución es necesario que exista traba entre ladrillos. Especial atención hay que poner en las distintas secciones que se producen conforme aumenta la altura, ya que el número y dimensión de piezas es distinto en cada hilada. Cada cierto número de hiladas, cada cierto tiempo, o al término de cada día, se limpiaban y rejuntaban las llagas y tendeles. Para ello se preparaba un mortero con arena más fina, se apretaba la junta con la paleta, usualmente con junta matada inferior, aunque no era lo más recomendable por la entrada de agua, o con una anilla (Figura 8a), como los murcianos, que se manejaba redibujando una línea en la junta y que es apreciable, hasta tal punto, que ha sido una de las características a tener en cuenta para establecer la autoría de algunas de las chimeneas encontradas en la zona de La Sagra. Mediante el sistema del llaguero con galga de madera provisto de un saliente para realizar la junta de manera rehundida ha realizado la operación del rejuntado la familia Cortés (Figura $8 \mathrm{~b}$ ). También rehundida queda la llaga con una pieza metálica curva que se adapta a la mano que utiliza la familia Alfonso (Figura 8c). Para limpiar las rebabas y sobrantes se repasaba el ladrillo con un fregal de esparto.

Con el fin de que el hueco interior no se estreche excesivamente para permitir el trabajo de los operarios, junto con la intención de aligerar el peso propio, se procede aproximadamente cada $5 \mathrm{~m}$, a la disminución por el interior de medio pie de ladrillo o el tamaño correspondiente a una cuña, propiciando la aparición de un escalonado interior, llegándose a utilizar durante la construcción de la chimenea, para el descanso durante el ascenso al lugar de trabajo (Figura 9). Riquelme afirma que estos resaltes eran matados con pelladas de mortero para facilitar el camino de salida de humos, mientras que el resto de entrevistados prefiere dejarlo vivo aduciendo que era fundamental para ayudar al tiro esta especie de corrientes que se formaban por el enfriamiento de los humos en estos pequeños recovecos.

Para la comprobación de la pendiente durante la construcción, se procedía al ejecutar cada hilada, en el caso de fus- 

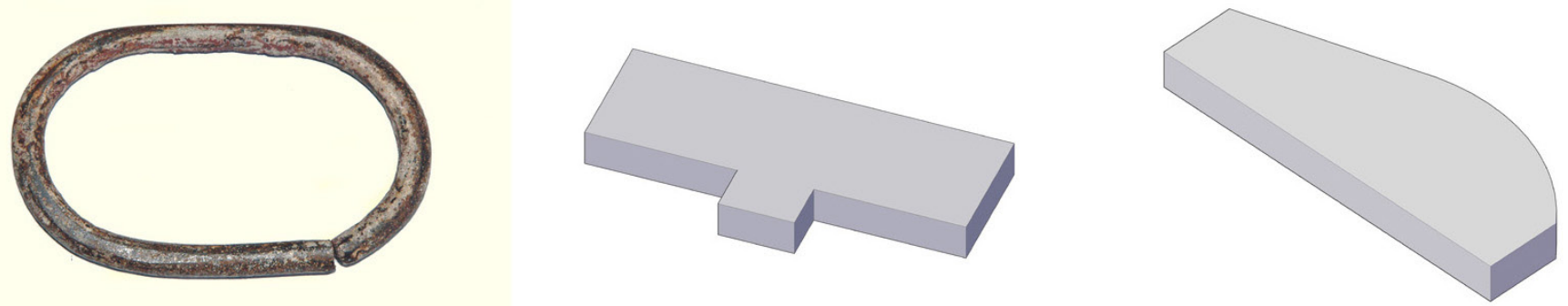

Figura 8. Piezas para rejuntar el mortero de juntas: a) anilla metálica; b) pieza en T; c) pieza metálica.

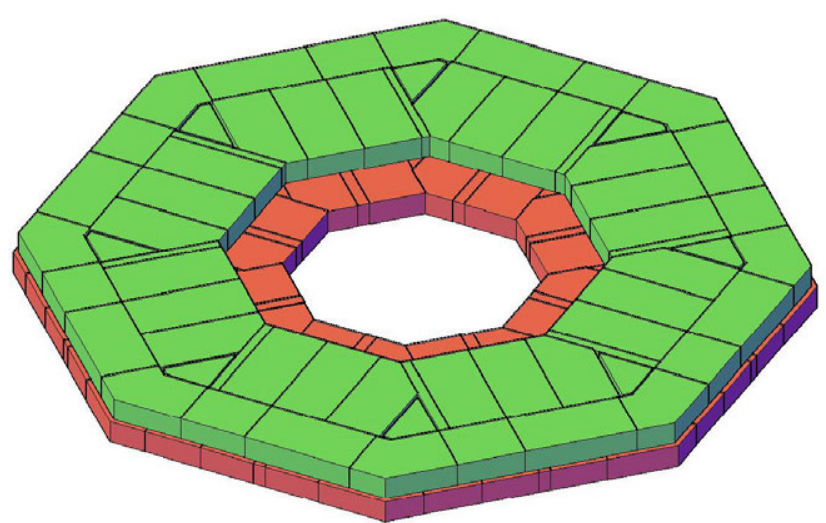

Figura 9. Montaje de una sección octogonal con piezas aplantilladas y escalón interior.

te octogonal, a su verificación en cada una de las caras del octógono por dos veces; es decir, un total de 16 veces. Para dicha operación se introducía el clavo sobresaliente del taulaplom, instrumento de madera de $1 \mathrm{~m}$ de longitud provisto de pendiente igual a la del fuste, entre las llagas del ladrillo para comprobar dicha pendiente. En su parte superior se colocaba un nivel de burbuja para conseguir la aplomada total (Figura 10).

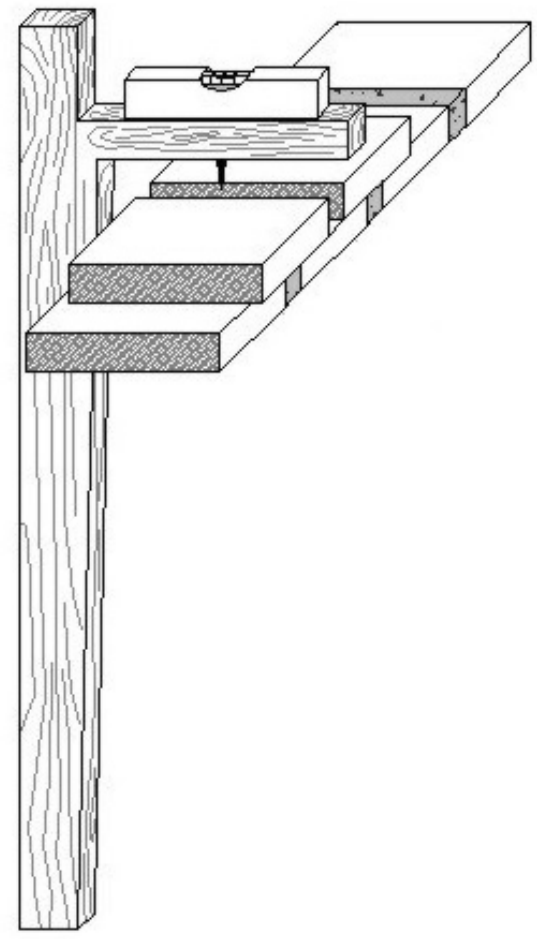

Figura 10. Taulaplom.
Para un fuste circular es suficiente con la comprobación de la circularidad con una gafa compás o cintrel cada ciertas hiladas y la pendiente con el taulaplom una sola vez por hilada.

Respecto a la policromía de cornisas y esquinas, varios eran los métodos para la coloración de los ladrillos. Los maestros valencianos introducían las piezas en un barreño con almagra y vinagre, que tiene la misión de fijar el color. Primero bañaban un lado, luego el otro, mientras que los murcianos colorean una vez colocados los ladrillos, cada tres o cuatro hiladas, sin utilización del vinagre.

Para ascender hasta el lugar de trabajo se disponen anillas metálicas cada 7-9 hiladas, es decir, cada $50 \mathrm{~cm}$. Las anillas tienen forma de $\mathrm{U}$ o [, con los extremos abiertos en forma de garra. Se coloca la anilla entre las hiladas de manera alineada con las inferiores, caso del vapor Aymerich en Terrassa, o de manera alternada a izquierda y derecha, como en la chimenea de Casinos, dentro de la misma pared vertical, para facilitar la ascensión y apoyo de cada pie. Como caso curioso, Jareño utiliza barras rectas colocadas en las esquinas, pero en número de dos, de manera que, además de servir para el ascenso, eran utilizadas para apoyar el andamiaje. De las Rivas advierte del peligro que para la fábrica puede suponer la dilatación lineal del hierro y aconseja temperatura de humos por debajo de los $300^{\circ}$. Menos afectadas por la temperatura serían aquellas que disponen de una escala exterior. Existen tres variantes, una con anillas metálicas de igual tamaño en forma de [, como la de la Figura 11, o de dos tamaños distintos, siendo una de ellas más grande que la otra y otras que combinan las anteriores con otras en forma de C, para aumentar la seguridad, como en el ejemplo de la chimenea Saphil de Terrassa.

Para finalizar la construcción, realizada la corona, se procedía a cerrar el hueco con la boquilla formada por 6-8 hiladas (Figura 12), que para chimeneas de alturas superiores a los 25-30 m se realizan de un pie de espesor, mientras que por debajo de esta altura el espesor es de medio pie. Constructores como los Martínez enrollaban varias vueltas de alambre de acero fino alrededor de esta boquilla, tapándolo con una capa de mortero de cemento que dejaban con forma convexa, y que luego podían revestir de trencadís cerámico.

Lo último en ejecutarse es el conducto de humos, que puede o no coincidir con el hueco de acceso para la ejecución de la obra. Para ello ejecuta el abovedado con un sistema parecido al empleado en la abertura de la chimenea.

\subsection{Pararrayos}

La colocación de un pararrayos se hacía imprescindible en estas construcciones. Su montaje ya se inicia mientras se rea- 


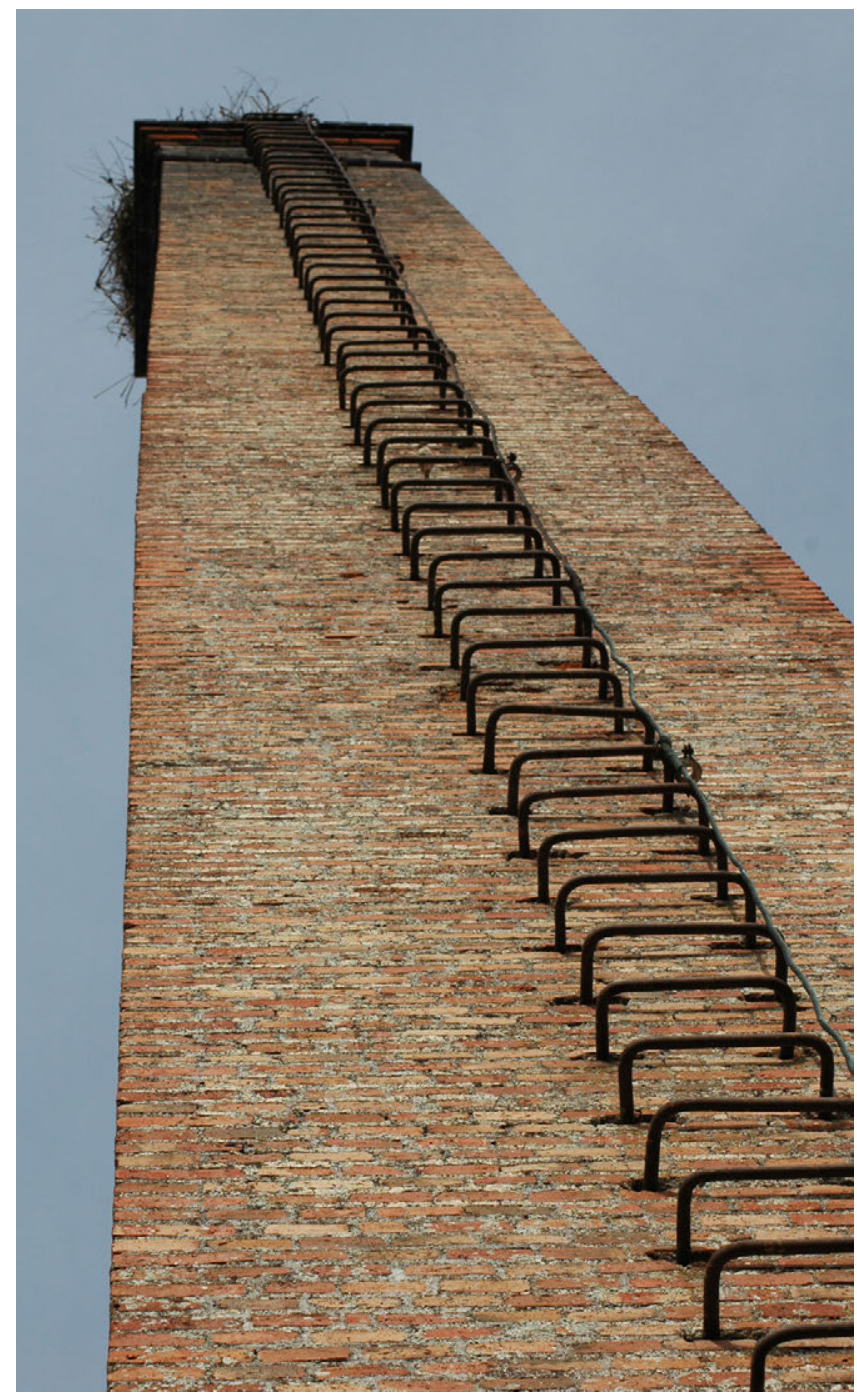

Figura 11. Anillas exteriores en forma de [ de la chimenea Electro Harinera Panificadora. Almodóvar del Campo (Ciudad Real).

liza la fábrica de ladrillo, colocando los aisladores entre 1,50 y 2,50 m. Con cada hilada se debía recordar arrastrar el cable para evitar problemas posteriormente. Cuando ya se ha construido la boquilla se procede a la colocación del pararrayos. Se iza entonces el cable que conectará el pararrayos con la tierra. Se ata el cable o cuerda que anteriormente había pasado mediante aguja por los aisladores y se estira de él hasta que llega a la cumbrera. Se fija entonces al pararrayos con garras. Los Jareño izan el cable por el interior y lo dejan caer desde arriba, estirando de él desde abajo, de manera que no han de arrastrar el peso.

La última operación en ejecutarse es la colocación de la toma a tierra. Para ello Salvador Mir detalla la operación tal como sigue:

se practica un hueco en el suelo de al menos $1 \times 1 \times 1 \mathrm{~m}$. Excavada la tierra se procede a rellenar con unos $20 \mathrm{~cm}$ de carbón piedra, unos tres dedos de sal gruesa, se coloca la placa metálica a la que previamente se le habrá soldado o atornillado el cable procedente del pararrayos. Se vuelve a colocar otra capa de carbón y tierra hasta enrasar, empapándolo todo muy bien, para que la transmisión de la corriente eléctrica sea adecuada.

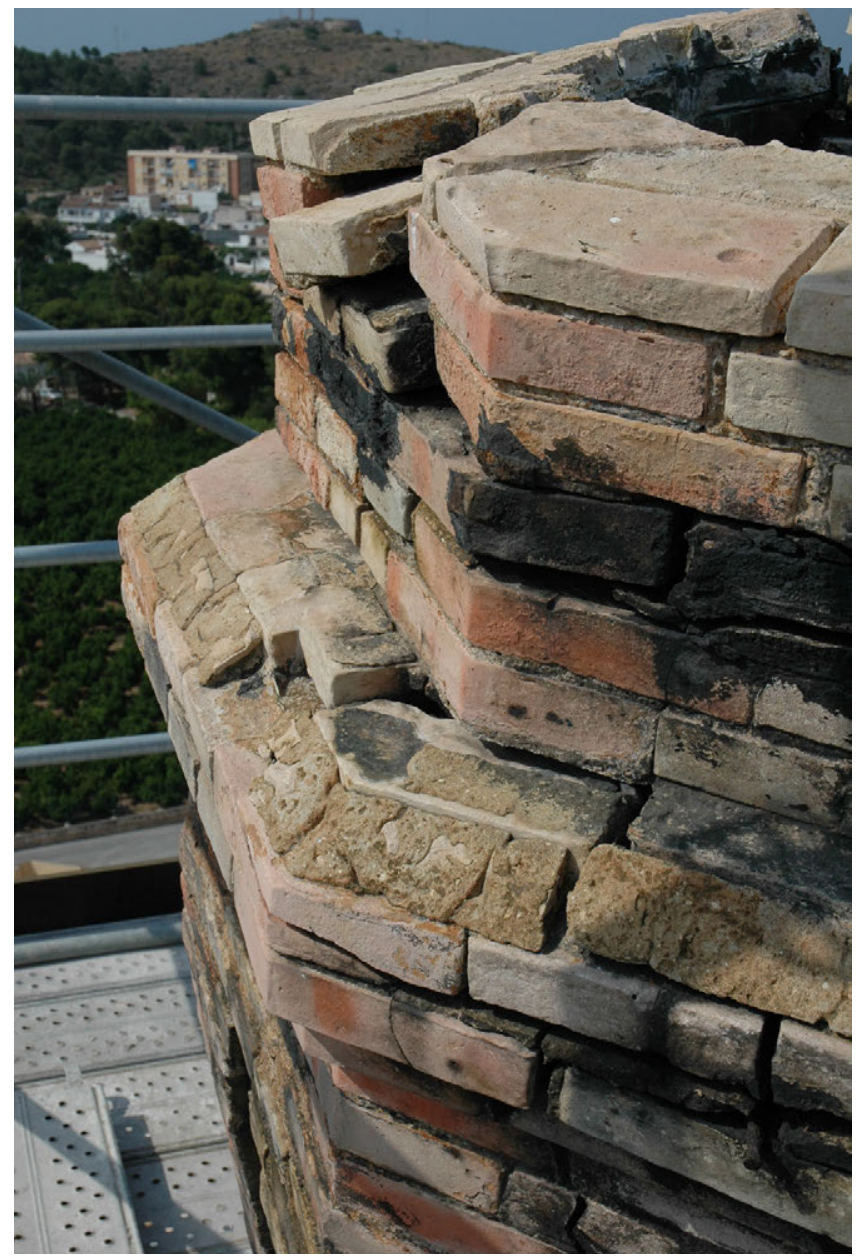

Figura 12. Corona de chimenea con bordón y boquilla.

\section{CONCLUSIONES}

Las chimeneas de ladrillo elevadas en la zona de levante responden, tanto por su geometría, ejecución como por medios auxiliares empleados, a la construcción por equipos murcianos o valencianos.

Los equipos valencianos requerían tan sólo de cinco personas para la ejecución de una chimenea industrial de fuste de sección transversal octogonal, con ayuda de una polea para transportar materiales por el interior de la misma y la utilización masiva del taulaplom, hasta 16 veces por hilada, para mantener la pendiente entre $2-3 \%$.

Los equipos murcianos están formados por ocho personas, con una disposición de polea por el exterior que hace posible el transporte, aunque con mayor cuidado para evitar golpes contra la superficie trabajada. Además del taulaplom, se ayudan de una gafa compás u otro sistema para comprobar que el centro de la sección circular trabajada corresponde con el centro ubicado en cota cero. Raramente colocan aisladores para el paso del cable de pararrayos.

De las diferentes geometrías y sistemas de construcción de las chimeneas estudiadas se extrae la mayor dificultad en la ejecución, diseño y cálculo de una chimenea octogonal, que necesita de piezas especiales, y de un paso de una geometría cuadrada en base a una octogonal en fuste, ejecutada originalmente por valencianos. 


\section{REFERENCIAS}

(1) Valdés, N. (1870). Manual del ingeniero y arquitecto. Madrid: Imprenta de Gabriel Alhambra.

(2) Rebolledo, J.A. (1875). Construcción general. Madrid: Imprenta y Fundición de J. Antonio García.

(3) Wilson, R. (1877). Boiler and factory chimneys, their draught-power and stability. London: Crosby Lockwood \& Co.

(4) Buchanan, R. (1850). An account of the chimney of the Edimburg Gas Works, with observations on the principles of its strenght and stability. Civil Engineers and Architects Journal.

(5) López Patiño, G. (2014). Chimeneas industriales de ladrillo en el levante y sureste español. Influencias sobre otros territorios. Estudio y análisis de las tipologías constructivas. Valencia: Editorial Universidad Politécnica València.

(6) Bancroft, R.M., Bancroft F.J. (1885). A practical treatise on the construction of Tall chimney Shafts. Manchester: John Calvert.

(7) Cascales López, P.L. (2001). Las chimeneas industriales de Alcantarilla. Alcantarilla: Ferretería Zapata.

(8) De las Rivas y López, M. (1905). Chimeneas de fábrica: teoría, cálculo de sus dimensiones, estabilidad, construcción. Madrid: [s.n.] Imprenta del Memorial de Ingenieros del Ejército. 